\title{
Symptoms and Upper Gastrointestinal Mucosal Injury Associated with Bisphosphonate Therapy
}

\author{
Kana Yamamoto, Maiko Kishino, Shinichi Nakamura and Katsutoshi Tokushige
}

\begin{abstract}
:
Objective The incidence of osteoporosis is increasing with the rapid aging of the Japanese population. Bisphosphonates are first-line agents used for the treatment of osteoporosis, but they can cause upper gastrointestinal mucosal injury. This study investigated symptoms and upper gastrointestinal mucosal injury associated with oral bisphosphonates.

Methods Symptoms were evaluated using the F-scale questionnaire, and esophageal mucosal injury and gastroduodenal ulceration were assessed by endoscopy. Patients were stratified by the type of bisphosphonate (alendronate, risedronate, or minodronate), treatment schedule (once weekly or every four weeks), and the concomitant use of other medications [antithrombotic agents, nonsteroidal anti-inflammatory drugs (NSAIDs), or acid suppressants].

Patients The subjects included 221 patients treated with oral bisphosphonates for at least one month.

Results The median F-scale total score was 4 (0-34), reflux score was $2(0-20)$, and the mean dyspepsia score was 2 (0-16). Endoscopy showed esophageal mucosal injury of Grade A or worse (Los Angeles classification) in 22/221 patients $(10.0 \%)$ and gastroduodenal ulcers in 9 patients $(4.1 \%)$. The dyspepsia score in patients who took minodronate every four weeks was significantly lower $(\mathrm{p}<0.05)$ in comparison to patients who took other bisphosphonates. The dyspepsia score was significantly higher $(\mathrm{p}<0.05)$ and mucosal injury was significantly more frequent in patients who also used antithrombotic agents and NSAIDs.

Conclusion Symptoms and upper gastrointestinal mucosal damage were not necessarily frequent or severe in patients treated with bisphosphonates. However, the concomitant use of bisphosphonates with antithrombotic agents and NSAIDs increased both symptoms and mucosal injury. The symptoms were milder in patients using minodronate once monthly.
\end{abstract}

Key words: bisphosphonates, endoscopy, F-scale, mucosal injury

(Intern Med 58: 1049-1056, 2019)

(DOI: 10.2169/internalmedicine.1271-18)

\section{Introduction}

There has been a marked increase in patients with osteoporosis in Japan due to the rapid aging of the population. It is currently estimated that there are 12.8 million such patients in the country ( 3 million men and 9.8 million women). Osteoporosis increases the risk of bone fracture, especially fracture of the vertebral bodies, the forearm bones, and the distal femur. Fractures not only reduce mobility and affect the activities of daily living, but also increase the chance of debilitating morbidities and mortality.
In Japan, the incidence of osteoporotic fractures has been increasing in the past 20 years and the prevention of osteoporosis is considered an important issue (1).

Osteoporosis is a condition characterized by diminished bone strength and a higher risk of fracture. Since the bone mineral density (BMD) accounts for $70 \%$ of the total bone strength, improvement of the BMD is the key to any treatment designed to improve bone strength in osteoporosis patients. Currently available drugs that increase BMD include bisphosphonates, female hormones, parathyroid hormone (PTH), selective estrogen receptor modulators (SERM), active vitamin D preparations, calcium preparations, calcitonin, 
and vitamin $\mathrm{K}$. Bisphosphonates are recommended as a firstline treatment for osteoporosis. However, bisphosphonate therapy can cause upper gastrointestinal mucosal injury, which leads to poor compliance (2). It is thought that the direct effect of bisphosphonates on the gastrointestinal mucosa leads to gastrointestinal tract damage and symptoms (3). By binding to the gastrointestinal mucosa, bisphosphonates replace hydrophobic and acid-resistant phospholipids involved in the mucosal barrier mechanism, and the weakening of the barrier leads to mucosal injury, such as erosion and ulceration (4). In some patients, bisphosphonate-induced mucosal injury is severe (5), and thus early detection and appropriate countermeasures are important.

Patients must be given detailed instructions on the use of oral bisphosphonates, and special attention must be paid to patients with impaired esophageal transit as well as patients with specific physical features (e.g., gibbus deformity). However, some patients without such conditions also develop mucosal injury. Thus, there is a need to identify the risk factors for mucosal injury associated with bisphosphonate therapy. The F-scale is a 12-item questionnaire used to evaluate acid reflux symptoms and dyspepsia symptoms; reflux esophagitis is suspected when the F-scale total score is $\geq 8$ points (6). In this study, we evaluated the symptoms of patients taking oral bisphosphonates using the F-scale questionnaire and investigated the association between the findings and the severity of upper gastrointestinal mucosal injury.

\section{Materials and Methods}

\section{Patients}

Among patients who visited the Department of Gastroenterology at our hospital between 2012 and 2016 and who underwent an evaluation of symptoms and upper gastrointestinal endoscopy, 221 women who had used an oral bisphosphonate (BP) for at least one month were enrolled in this study. Patients were eligible for enrollment if their symptoms had been evaluated using the Frequency Scale for the Symptoms of gastroesophageal reflux disease (GERD) (Fscale) questionnaire, with the total score, reflux score, and dyspepsia score being determined. Patients with advanced cancer of the upper gastrointestinal tract (including the esophagus, stomach, and duodenum) and patients with a history of upper gastrointestinal tract surgery were excluded from this study. Upper gastrointestinal endoscopy was performed as a routine screening test in all patients using an Olympus electronic endoscope (GIF-Q290 or GIF-H290, Olympus, Tokyo, Japan).

\section{Methods}

This retrospective observational study included patients who visited the Gastroenterology Department for other studies and who underwent upper gastrointestinal endoscopy as a routine screening test, as well as an F-scale evaluation.
This study was approved by the human ethics committee of Tokyo Women's Medical University. Along with the F-scale scores, the following data were extracted from the medical records: age, gender, use or non-use of oral BPs, type of BP, treatment schedule, concomitant drugs (oral antithrombotic agents, oral NSAIDs, and oral acid suppressants), Helicobacter pylori infection, and endoscopic findings (esophagitis, hiatal hernia, gastroduodenal ulcers, and atrophic gastritis). Endoscopic images were read and the diagnosis was made by a gastroenterologist who had been certified by the Japan Gastroenterological Endoscopy Society.

\section{Clinical assessments}

The grade of reflux esophagitis [Los Angeles classification (7)], the presence of gastroduodenal erosion/ulcer, and the severity of background gastric mucosal atrophy (8) and hiatus hernia (9) were evaluated by endoscopic examination in all 221 patients. For the evaluation of reflux esophagitis, patients were stratified according to the LA classification into those without mucosal injury (None group), patients with Grade A-D disease with mucosal damage (Grade A-D group), and patients with certain specific conditions such as desquamative esophagitis or esophageal submucosal hematoma (special group). The F-scale scores of these three groups were then compared. With regard to gastroduodenal ulceration, patients were classified into those with and without gastroduodenal ulcers. The F-scale scores were compared between the two groups. In this study, a gastroduodenal ulcer was defined by the presence of $\mathrm{H}_{2}$ or more advanced disease, according to the Sakita-Miwa classification (10).

The patients were classified into three groups based on the type of bisphosphonate used for treatment: the alendronate group, risedronate group, and minodronate group. The F-scale scores were compared among the three groups. The frequency of upper gastrointestinal mucosal injury was also compared among the groups. In this study, upper gastrointestinal mucosal injury was defined as Grade A or more severe esophagitis (according to the LA classification) or the presence of gastroduodenal ulcers. Furthermore, the patients were classified into two groups based on the treatment schedule: the weekly group (treated with alendronate or risedronate once per week) and the monthly group (treated with minodronate every four weeks). The F-scale scores and the frequency of upper gastrointestinal mucosal injury in the two groups were compared.

Patients were also classified into four groups based on concomitant antithrombotic agents: the antithrombotic group, the NSAIDs group, the antithrombotic + NSAIDs group, and the drug non-use group (patients who did not use antithrombotic agents or NSAIDs). The F-scale scores and frequency of upper gastrointestinal mucosal damage were compared among these groups.

Finally, the patients were classified into two groups based on use or non-use of acid-suppressing agents: the acidsuppressing agent group and the acid-suppressing agent nonuse group. The F-scale scores and the incidence of upper 
gastrointestinal mucosal damage were compared between these two groups.

\section{Statistical analysis and ethical considerations}

The results are shown as the median value or percentage. Comparisons between two groups (see Table 1-3) were performed by using the Mann-Whitney test, while comparisons among three or four groups (see Table 4-6) were performed with the Kruskal Wallis test. $\mathrm{P}$ values of $<0.05$ were considered to indicate a statistically significant difference. When the F-scale total score was $\geq 8$ points, the patient was classified as having symptoms. The percentage of patients with symptoms was compared among groups using the chisquared test. All analyses were performed using the JMP pro 13 software program.

This study conformed to the ethical guidelines of the

Table 1. The F-scale Scores of Patients Treated with Bisphosphonates according to the Presence or Absence of Gastroduodenal Ulceration.

\begin{tabular}{lccc}
\hline \multicolumn{1}{c}{ FSSG } & $\begin{array}{c}\text { Non-ulcer group } \\
(\mathrm{n}=212)\end{array}$ & $\begin{array}{c}\text { Ulcer group } \\
(\mathrm{n}=9)\end{array}$ & p value \\
\hline Total score & $4(0-34)$ & $4(0-26)$ & $\mathrm{NS}$ \\
$\geq 8$ points $(\%)$ & $67 / 212(31.6)$ & $3 / 9(33.3)$ & $\mathrm{NS}$ \\
Reflux score & $2(0-20)$ & $2(0-10)$ & $\mathrm{NS}$ \\
Dyspepsia score & $2(0-16)$ & $2(0-16)$ & $\mathrm{NS}$ \\
\hline
\end{tabular}

Data are expressed as the number of patients (\%) or median (minimummaximum).

Statistical comparisons were performed using the Wilcoxon test or chisquared test.
Declaration of Helsinki (2000 revision) and was approved by the ethics committee of Tokyo Women's Medical University. We obtained informed consent for the study directly when we could contact the patients. In addition, we published the study plan on our hospital's home page. If a patient or a bereaved family member disapproved of the patient's data being used in this study, we deleted the relevant data.

The authors declare no conflicts of interest in association with the present study.

\section{Results}

Bisphosphonates were used for the treatment of osteoporosis $(n=104)$, prevention of secondary osteoporosis in patients on oral prednisolone $(n=93)$, and secondary osteoporosis due to renal dysfunction or endocrine diseases $(n=24)$. All patients were women. The mean age was 71 (34-89) years. The bisphosphonates used by the patients were alendronate $(n=124)$, risedronate $(n=66)$, and minodronate $(n=$ 31). Alendronate and risedronate were administered once per week in 190 patients, while minodronate was administered every four weeks in 31 patients. The duration of oral BP administration was $<3$ months in 7 patients, 3-12 months in 9 patients, and $>12$ months in 205 patients. Concomitant drugs included prednisolone $(\mathrm{n}=93)$, nonsteroidal antiinflammatory drugs (NSAIDs) $(n=50)$, and antithrombotic agents $(n=42)$ [low-dose aspirin $(n=18)$, warfarin $(n=20)$, clopidogrel bisulfate $(n=7)$, and others $(n=6)$; some patients

Table 2. The F-scale Scores in Patients Treated with Bisphosphonates according to the Frequency of and Bisphosphonate Administration.

\begin{tabular}{lccc}
\hline \multicolumn{1}{c}{ FSSG } & $\begin{array}{c}\text { Weekly group } \\
(\mathrm{n}=190)\end{array}$ & $\begin{array}{c}\text { Monthly group } \\
(\mathrm{n}=31)\end{array}$ & $\mathrm{p}$ value \\
\hline Total score & $4(0-34)$ & $2(0-21)$ & 0.0172 \\
$\geq 8$ points $(\%)$ & $64 / 190(33.7)$ & $6 / 31(19.4)$ & $\mathrm{NS}$ \\
Reflux score & $2(0-20)$ & $1(0-12)$ & $\mathrm{NS}$ \\
Dyspepsia score & $2(0-16)$ & $0(0-11)$ & 0.0045 \\
Frequency of mucosal injury & $29 / 190(15.3)$ & $2 / 31(6.5)$ & $\mathrm{NS}$ \\
\hline
\end{tabular}

Data are expressed as the number of patients (\%) or median (minimum-maximum). Statistical comparisons were performed using the Wilcoxon test or chi-squared test.

Table 3. The F-scale Scores of Patients Treated with Bisphosphonates according to the Administration or Non-administration of Acid-suppressing Agents.

\begin{tabular}{lccc}
\hline \multicolumn{1}{c}{ FSSG } & $\begin{array}{c}\text { Acid-suppressing agent } \\
\text { group }(\mathrm{n}=149)\end{array}$ & $\begin{array}{c}\text { Acid-suppressing agent } \\
\text { non-use group (n=72) }\end{array}$ & p value \\
\hline Total score & $4(0-28)$ & $4(0-34)$ & NS \\
$\geq 8$ points $(\%)$ & $44 / 149(29.5)$ & $26 / 72(36.1)$ & NS \\
Reflux score & $2(0-20)$ & $2(0-18)$ & NS \\
Dyspepsia score & $2(0-15)$ & $2(0-16)$ & NS \\
Frequency of mucosal injury & $17 / 149(11.4)$ & $14 / 72(19.4)$ & NS \\
\hline
\end{tabular}

Data are expressed as the number of patients (\%) or median (minimum-maximum).

Statistical comparisons were performed using the Wilcoxon test or chi-squared test. 
Table 4. The F-scale Scores in Patients Treated with Bisphosphonates according to the Presence or Absence of Esophagitis.

\begin{tabular}{lcccc}
\hline \multicolumn{1}{c}{ FSSG } & $\begin{array}{c}\text { None group } \\
(\mathrm{n}=197)\end{array}$ & $\begin{array}{c}\text { Grade A-D group } \\
(\mathrm{n}=22)\end{array}$ & $\begin{array}{c}\text { Special group* } \\
(\mathrm{n}=2)\end{array}$ & $\mathrm{p}$ value \\
\hline Total score & $4(0-26)$ & $8.5(0-34)$ & $14.5(11-19)$ & 0.0179 \\
$\geq 8$ points $(\%)$ & $56 / 197(28.4)$ & $12 / 22(54.5)$ & $2 / 2(100)$ & 0.0050 \\
Reflux score & $2(0-20)$ & $5(0-18)$ & $4.5(1-8)$ & 0.0107 \\
Dyspepsia score & $2(0-16)$ & $2(0-16)$ & $10.0(9-11)$ & 0.0457 \\
\hline
\end{tabular}

Data are expressed as the number of patients (\%) or median (minimum-maximum).

Statistical comparisons were performed using the Kruskal Wallis test or chi-squared test.

*Submucosal hematoma and erosive esophagitis.

Table 5. The F-scale Scores according to the Type of Bisphosphonate Used for Treatment.

\begin{tabular}{lcccc}
\hline \multicolumn{1}{c}{ FSSG } & $\begin{array}{c}\text { Alendronate group } \\
(\mathrm{n}=124)\end{array}$ & $\begin{array}{c}\text { Risedronate group } \\
(\mathrm{n}=66)\end{array}$ & $\begin{array}{c}\text { Minodronate group } \\
(\mathrm{n}=31)\end{array}$ & $\mathrm{p}$ value \\
\hline Total score & $4(0-34)$ & $5(0-24)$ & $2(0-21)$ & 0.0361 \\
$\geq 8$ points $(\%)$ & $38 / 124(30.6)$ & $26 / 66(39.4)$ & $6 / 31(19.4)$ & $\mathrm{NS}$ \\
Reflux score & $2(0-20)$ & $2(0-15)$ & $1(0-12)$ & $\mathrm{NS}$ \\
Dyspepsia score & $2(0-16)$ & $2(0-10)$ & $0(0-11)$ & 0.0088 \\
Frequency of mucosal injury & $21 / 124(16.9)$ & $8 / 66(12.1)$ & $2 / 31(6.5)$ & $\mathrm{NS}$ \\
\hline
\end{tabular}

Data are expressed as the number of patients (\%) or median (minimum-maximum).

Statistical comparisons were performed using the Kruskal Wallis test or chi-squared test.

Table 6. The F-scale Scores of Patients Treated with Bisphosphonates according to the Administration of Other Medications.

\begin{tabular}{|c|c|c|c|c|c|}
\hline FSSG & $\begin{array}{l}\text { Anti-thrombotic } \\
\text { group }(n=30)\end{array}$ & $\begin{array}{l}\text { NSAIDs group } \\
\quad(n=38)\end{array}$ & $\begin{array}{c}\text { Anti-thrombotict } \\
\text { NSAIDs group }(n=12)\end{array}$ & $\begin{array}{l}\text { Drug non-use group } \\
\qquad(\mathrm{n}=141)\end{array}$ & $\mathrm{p}$ value \\
\hline Total score & $3(0-14)$ & $4(0-24)$ & $9(1-20)$ & $4(0-34)$ & 0.0501 \\
\hline$\geq 8$ points $(\%)$ & $8 / 30(26.7)$ & $13 / 38(34.2)$ & $7 / 12(58.3)$ & $42 / 141(29.8)$ & NS \\
\hline Reflux score & $0(0-11)$ & $3(0-14)$ & $4(0-13)$ & $2(0-20)$ & NS \\
\hline Dyspepsia score & $1(0-11)$ & $2(0-11)$ & $6(1-11)$ & $1(0-16)$ & 0.0191 \\
\hline Frequency of mucosal injury & $3 / 30(10.0)$ & $5 / 38(13.2)$ & $5 / 12(41.7)$ & $18 / 141(12.8)$ & 0.0418 \\
\hline
\end{tabular}

Data are expressed as the number of patients (\%) or median (minimum-maximum).

Statistical comparisons were performed using the Kruskal-Wallis test or chi-squared test.

were on more than one of these drugs]. In addition, the patients were treated with acid suppressants, including proton pump inhibitors (PPIs) $(\mathrm{n}=108)$ and $\mathrm{H}_{2}$-blockers $(\mathrm{n}=41)$. Forty-four patients underwent testing for Helicobacter pylori infection; the results were positive in 13 patients (Table 7).

\section{Symptoms}

In all 221 patients, the median F-scale total score was 4 (0-34) points. A total score of $\geq 8$ points, suggestive of reflux esophagitis/GERD, was recorded in 70 (31.7\%) patients. On the other hand, the median F-scale reflux score was $2(0-20)$ points and the median dyspepsia score was $2(0-16)$ points.

\section{The relationship between the upper gastrointestinal} endoscopic findings and symptoms

Endoscopic findings: Endoscopy showed reflux esophagitis without mucosal injury in $197(89.1 \%)$ patients, while the classification was Grade A in $12(5.4 \%)$, Grade B in 7
(3.2\%), Grade C in $2(0.9 \%)$, and Grade D in $1(0.5 \%)$. Esophageal submucosal hematoma (1 patient, $0.5 \%)$ and desquamative esophagitis (1 patient, $0.5 \%$ ) were also detected. Furthermore, $9(4.1 \%)$ patients were found to have multiple gastroduodenal ulcers. On the other hand, 108 (48.9\%) patients were found to have no background gastric mucosal atrophy, while 47 (21.3\%) and 66 (29.9\%) patients had mild and severe atrophy, respectively. Interestingly, 69 patients (31.2\%) were found to have hiatal hernia (Table 8).

Reflux esophagitis: The median F-scale total score was 4 $(0-26), 8.5(0-34)$, and $14.5(10-19)$ points in the None $(n=$ 197), Grade A-D ( $\mathrm{n}=22)$, and special $(\mathrm{n}=2)$ groups, respectively. In addition, the median F-scale reflux scores were 2 $(0-20), 5$ (0-18), and 4.5 (1-8) points, respectively, while the median F-scale dyspepsia scores were 2 (0-16), 2 (0-16), and 10 (9-11) points, respectively (Table 4). The comparison of the three groups showed that the percentage of patients with an F-scale total score of $\geq 8$ points was significantly 
Table 7. The Baseline Characteristics of the Patients Treated with Bisphosphonates.

\begin{tabular}{|c|c|}
\hline Characteristic & All patients $(n=221)$ \\
\hline Age (years) & $71(34-89)$ \\
\hline Gender (Male/Female) & $0 / 221$ \\
\hline Helicobacter pylori positive & $13 / 44(29.5)$ \\
\hline \multicolumn{2}{|l|}{ Bisphosphonate therapy } \\
\hline Alendronate & 124 \\
\hline Risedronate & 66 \\
\hline Minodronate & 31 \\
\hline \multicolumn{2}{|l|}{ Frequency } \\
\hline Weekly & 190 \\
\hline Monthly & 31 \\
\hline \multicolumn{2}{|l|}{ Duration of BP therapy } \\
\hline$<3$ months & 7 \\
\hline 3 12 months & 9 \\
\hline$>12$ months & 205 \\
\hline \multicolumn{2}{|l|}{ Other medications } \\
\hline Anti-thrombotic agents & 42 \\
\hline Aspirin & 18 \\
\hline Warfarin & 20 \\
\hline Clopidogrel & 7 \\
\hline NSAIDs & 50 \\
\hline Corticosteroids & 93 \\
\hline Acid-suppressing agents & 149 \\
\hline Proton pump inhibitors & 108 \\
\hline $\mathrm{H} 2$ antagonists & 41 \\
\hline
\end{tabular}

Data are expressed as the number of patients $(\%)$ or median (minimum-maximum).

NSAIDs: nonsteroidal anti-inflammatory drugs

larger in the special group $(\mathrm{p}=0.0050)$. In addition, the Fscale reflux score was significantly higher in the Grade A-D group $(\mathrm{p}=0.0107)$ while the dyspepsia score was significantly higher in the special group $(\mathrm{p}=0.0457)$.

Gastroduodenal ulcers: The median F-scale total score was 4 (0-26) points in the 9 patients with ulcers (ulcer group) and 4 (0-34) points in the 212 patients without ulcers (non-ulcer group). There were no significant differences between the two groups with regard to the F-scale total score, reflux score, or dyspepsia score.

\section{Symptoms associated with bisphosphonates}

Types of bisphosphonates: The median F-scale total scores were $4(0-34), 5(0-24)$, and $2(0-21)$ points in the alendronate group $(n=124)$, risedronate group $(n=66)$, and minodronate group $(n=31)$, respectively. In addition, the median F-scale reflux scores were 2 (0-20), 2 (0-15), and 1 (012) points, respectively, while the median dyspepsia scores were $2(0-16), 2(0-10)$, and $0(0-11)$ points, respectively (Table 5). The total score and the dyspepsia score were both significantly lower in the minodronate group ( $\mathrm{p}=0.0361$ and $\mathrm{p}=0.0088$, respectively). The rates of upper gastrointestinal mucosal injury in the alendronate, risedronate, and minodronate groups were $16.9 \%, 12.1 \%$, and $6.5 \%$, respectively, with no significant differences among the three groups.

Treatment schedule: The median F-scale total score was
Table 8. The F-scale Scores and Endoscopic Findings of Patients Treated with Bisphosphonates.

\begin{tabular}{|c|c|}
\hline FSSG & \\
\hline Total score & $4(0-34)$ \\
\hline$\geq 8$ points & $70 / 221(31.7)$ \\
\hline Reflux score & $2(0-20)$ \\
\hline Dyspepsia score & $2(0-16)$ \\
\hline \multicolumn{2}{|l|}{ Endoscopic findings } \\
\hline \multicolumn{2}{|l|}{ Esophagus findings } \\
\hline \multicolumn{2}{|l|}{ Reflux esophagitis } \\
\hline None & $197(89.1)$ \\
\hline Grade A & $12(5.4)$ \\
\hline Grade B & $7(3.2)$ \\
\hline Grade C & $2(0.9)$ \\
\hline Grade D & $1(0.5)$ \\
\hline Submucosal hematoma & $1(0.5)$ \\
\hline Erosive esophagitis & $1(0.5)$ \\
\hline \multicolumn{2}{|l|}{ Gastroduodenal findings } \\
\hline Ulcer & $9(4.1)$ \\
\hline Erosion & $37(16.7)$ \\
\hline \multicolumn{2}{|l|}{ Hiatal hernia } \\
\hline Grade 0 & $152(68.8)$ \\
\hline Grade C & $55(24.9)$ \\
\hline Grade B & $10(4.5)$ \\
\hline Grade A & $4(1.8)$ \\
\hline \multicolumn{2}{|l|}{ Atrophic gastritis } \\
\hline None & $108(48.9)$ \\
\hline Closed type & $47(21.3)$ \\
\hline Open type & $66(29.9)$ \\
\hline
\end{tabular}

Data are expressed as the number of patients (\%) or median (minimum-maximum).

$4(0-34)$ points in the weekly group (using alendronate or risedronate once per week) $(\mathrm{n}=190)$ and $2(0-21)$ points the monthly group (receiving minodronate every four weeks) $(\mathrm{n}=31)$. In addition, the median F-scale reflux scores were 2 $(0-20)$ and 1 (0-12) points, respectively, while the median dyspepsia scores were $2(0-16)$ and $0(0-11)$ points, respectively (Table 2). Both the total and dyspepsia scores were significantly lower in the monthly group $(\mathrm{p}=0.0172$ and $\mathrm{p}=$ 0.0045 , respectively). The rate of upper gastrointestinal mucosal injury in the weekly (15.3\%) and monthly (6.5\%) groups did not differ to a statistically significant extent.

\section{Symptoms associated with concomitant drugs}

Antithrombotic agents and NSAIDs: antithrombotic agents were used by only 30 patients (antithrombotic group), NSAIDs were used by 38 patients (NSAIDs group), and both antithrombotic agents and NSAIDs were used by 12 patients (antithrombotic + NSAIDs group). One hundred forty-one patients used neither antithrombotic agents nor concomitant drugs (drug non-use group). The median Fscale total score was 3 (0-19), 4 (0-24), 9 (1-20), and 4 (034) points in the antithrombotic, NSAIDs, antithrombotic + NSAIDs, and drug non-use groups, respectively. In addition, the median F-scale reflux scores were 0 (0-11), 3 (0-14), 4 
(0-13), and $2(0-20)$ points, respectively, while the median dyspepsia scores were 1 (0-11), 2 (0-11), 6 (1-11), and 1 (016) points, respectively (Table 6). The median F-scale total score was higher in the antithrombotic + NSAIDs group $(\mathrm{p}=$ $0.0501)$ and the dyspepsia score was significantly higher in this group ( $\mathrm{p}=0.0191)$. Upper gastrointestinal mucosal injury was detected in $10.0 \%$ of the patients in the antithrombotic group, $13.2 \%$ of the patients in the NSAIDs group, $41.7 \%$ of the patients in the antithrombotic + NSAIDs group, and $12.8 \%$ of the patients in the drug non-use group, with the frequency being significantly higher in the antithrombotic + NSAIDs group ( $\mathrm{p}=0.0418)$.

Acid-suppressing agents: The median F-scale total score was $4(0-28)$ points in 149 patients who used acidsuppressing agents (acid-suppressing agent group) and 4 (034) points in 72 patients who did not use these agents (acidsuppressing agent non-use group) (Table 3). The total score, reflux score, and dyspepsia score of the two groups did not differ to a statistically significant extent. Upper gastrointestinal mucosal injury was detected in $11.4 \%$ of the patients in the acid-suppressing agents group and $19.4 \%$ of the patients in the acid-suppressing agent non-use group; the difference was not statistically significant.

\section{Discussion}

The incidence of osteoporosis is high among elderly women. In addition, it has been reported that the F-scale score differs between men and women, and that the F-scale questionnaire more frequently detects symptoms in women than in men $(11,12)$; thus, only women were enrolled in this study. The reasons for visiting hospital varied among the patients, with some being asymptomatic and others presenting for the assessment of symptoms. Thus, there may have been some bias in the study population.

In the 221 patients investigated in this study, the median F-scale total score was $4(0-34)$ points and $70(31.7 \%)$ patients had a total score $\geq 8$ points. Upper gastrointestinal endoscopy identified Grade A or more severe reflux esophagitis in $22(10.0 \%)$ patients and gastroduodenal ulcers in 9 (4.1\%) patients. Matsuki et al. (13) assessed the F-scale scores of 886 healthy subjects. They reported that the total score was $5.08 \pm 4.90$ points and that a total score of $\geq 8$ points was recorded in $33.2 \%$ of the subjects, while Grade A or more severe reflux esophagitis was assessed in $15.6 \%$ of the subjects. Yamamichi et al. (12) conducted a crosssectional study of 19,864 healthy adults and reported that the mean F-scale total score was $4.8 \pm 5.2$ points. Okamoto et al. (14) studied 8,031 subjects and found that the incidence of Grade A or more severe reflux esophagitis was $14.9 \%$. Takeshita et al. (11) reported that the incidence of Grade A or more severe reflux esophagitis was $11.3 \%$ while the incidence of gastroduodenal ulcers was $1.5 \%$. In comparison to these healthy subjects, the F-scale score was slightly higher among our patients using oral bisphosphonates. However, there was no difference in the proportion of subjects with a total score of $\geq 8$ points, and there were no marked differences in the prevalence of Grade A or more severe reflux esophagitis and gastroduodenal ulcers. Based on these findings, it seems that oral bisphosphonate therapy does not necessarily cause symptoms or increase the frequency or severity of upper gastrointestinal mucosal injury.

In this study, the endoscopic evaluation of reflux esophagitis and gastroduodenal ulcers was performed. The F-scale reflux score was significantly higher in patients with Grade A-D reflux esophagitis and the dyspepsia score was significantly higher in the special group. Acid reflux is one of the underlying causes of esophageal mucosal injury. However, the significantly higher dyspepsia score in the special group suggested that esophageal retention of bisphosphonates due to impaired esophageal motility may cause severe local damage (desquamative esophagitis and esophageal submucosal hematoma). There was no significant difference in the F-scale scores between the groups classified on the basis of gastroduodenal damage, probably because the gastric mucosal injury is not only associated with the direct effects of bisphosphonates but also with other factors (e.g., the bisphosphonate dose and gastric $\mathrm{pH}$ ).

The examination of the relationship between the type of bisphosphonates and the F-scale scores or the frequency of mucosal injury showed that there were no significant differences in the F-scale scores between the alendronate and the risedronate groups. However, the F-scale total score and the dyspepsia scores were significantly lower in patients treated with minodronate, which is administered every four weeks instead of weekly. On the other hand, there was no significant difference in the rate of mucosal injury between the two groups. One major reason for these differences may be that minodronate is administered every four weeks instead of weekly. Another study (15) compared the extent of upper gastrointestinal damage between patients taking oral risedronate at a dose of $2.5 \mathrm{mg}$ once per day and patients taking oral risedronate at a dose of $17.5 \mathrm{mg}$ once per week; they reported that the frequency of mucosal injury was significantly lower in the weekly administration group. Hagino et al. (16) compared the frequency of upper gastrointestinal damage between patients taking oral risedronate $(2.5 \mathrm{mg})$ once per day (the daily group) and patients taking oral risedronate $(70 \mathrm{mg}$ ) every four weeks (the monthly group), they reported a significantly lower rate in the monthly group. It is thought that approximately 5 days are required for the regeneration of the gastrointestinal tract epithelium, suggesting that the gastric mucosal injury caused by bisphosphonates theoretically heals within approximately one week. Thus, the gastrointestinal damage associated with severe symptoms may be less frequent in patients taking oral bisphosphonates once per week or every four weeks (17). In the present study, the F-scale total score and dyspepsia score were both lower and symptoms were milder in patients treated with minodronate (administered every four weeks) in comparison to patients using alendronate or risedronate (administered once per week). This difference may be related to the longer 
interval between doses, which provides a better chance for mucosal injury to heal (if it has occurred); thus, the symptoms would become milder. Amagase et al. (18) compared the injurious effects of three BPs (alendronate, risedronate, and minodronate) on the mucosa in rats, and reported that risedronate caused the least mucosal damage. However, there has been no previous comparison of these three BPs in humans. In the present study, no significant differences were observed among the three drugs.

We also evaluated the relationship between the use of other medications with bisphosphonates on the F-scale score and rate of mucosal injury. It has been reported that the irritant effect of bisphosphonates is weaker than that of NSAIDs or aspirin $(19,20)$. However, the concomitant use of bisphosphonates with these drugs can lead to the exacerbation of mucosal damage (21). In this study, the patients were classified into four groups based on the types of drugs that were used (the antithrombotic group, the NSAIDs group, the antithrombotic + NSAIDs group, and the drug non-use group). The comparison of these four groups showed that the F-scale dyspepsia score was significantly higher and mucosal injury was significantly more frequent in the antithrombotic + NSAIDs group than in the other groups. This finding suggests that the concomitant use of either antithrombotic agents or NSAIDs with bisphosphonates does not worsen the severity of symptoms or the frequency of mucosal damage, whereas the use of both antithrombotic agents and NSAIDs with bisphosphonates significantly increases symptoms and the frequency of mucosal damage. Gastric mucosal injury is not only associated with the direct effect of bisphosphonates, but is also influenced by other factors, including the gastric $\mathrm{pH}$. In patients with high dyspepsia scores, the retention of bisphosphonates in the esophagus or gastroduodenal region due to impaired motility may be a major factor responsible for the aggravation of mucosal injury and symptoms.

The F-scale scores of patients treated with or without the use of acid-suppressing agents did not differ to a statistically significant extent. The $\mathrm{pH}$ level of the stomach plays an important role in the development of gastric mucosal damage and the suppression of acid production can protect against such damage. However, some studies have shown that the injurious effects of bisphosphonates are stronger in a neutral $\mathrm{pH}$ environment $(\mathrm{pH} 7.0)$ than in an acidic environment $(\mathrm{pH}$ 4.0) $(22,23)$. Thus, while acid-suppressing agents can inhibit the injurious effect of gastric acid, these drugs may promote the injurious effect of bisphosphonates. It is possible that this interaction explains why the use or non-use of acid-suppressing agents had no significant effect in this study.

Because the special group $(n=2)$ may have had increased F-scale scores, the remaining 219 patients were compared after excluding the special group. However, the results of this sub-analysis did not differ from those of the original analysis of 221 patients.

In this study, the oral administration of bisphosphonates did not worsen the F-scale scores or exacerbate the symptoms of most patients; however, the concomitant use of bisphosphonates with both NSAIDs and antithrombotic agents was associated with the exacerbation of symptoms and a higher frequency of mucosal injury. It is suggested that impaired gastrointestinal motility prolongs the retention of bisphosphonates in the digestive tract and thus exacerbates mucosal damage and symptoms. Many patients taking oral bisphosphonates have spinal deformity due to osteoporosis, which increases the risk of retention of drugs in the digestive tract. Bisphosphonates are not only used to treat primary osteoporosis but also administered to patients with secondary osteoporosis. Patients taking steroids often have an underlying disease associated with impaired gastrointestinal motility (e.g., scleroderma). Also, many patients require treatment with various medications for various complications and the concomitant use of certain drugs may have an injurious effect on the digestive tract mucosa that exacerbates mucosal damage. Thus, it is important to thoroughly understand the complications and the types of drugs used by individual patients when giving instructions about the appropriate oral use of bisphosphonates. Oral bisphosphonates should be administered with caution, especially in patients in whom impaired digestive tract motility is suspected and those treated with other drugs that have the potential to cause gastrointestinal mucosal injury. Interestingly, the Fscale scores were lowest and symptoms were mildest in the patients taking minodronate, which was administered every four weeks, unlike the other bisphosphonates, which were administered weekly. These findings suggest that it is also important to select the optimal medication for each patient.

\section{Conclusion}

In the present study, oral bisphosphonates therapy was not necessarily associated with gastrointestinal symptoms or mucosal injury. However, the concomitant use of bisphosphonates with both antithrombotic agents and NSAIDs was associated with more severe symptoms and a higher rate of mucosal damage, suggesting that attention is required when multiple oral drugs are used together. The symptoms were mildest in patients treated with minodronate every four weeks, indicating that it is important to select the optimum oral bisphosphonate for each patient.

\section{The authors state that they have no Conflict of Interest (COI).}

\section{Acknowledgement}

We thank Dr. Ichiro Ishikawa (Hachiman Ishikawa Internal Medicine Clinic, Department of Digestive Endoscopy, Tokyo Women's Medical University) for cooperation in the collection of cases for this study.

\section{References}

1. Orimo H; Japan Osteoporosis Society. The Japanese Guideline for Prevention and Treatment of Osteoporosis 2015. Life Science Pub- 
lishing Co., Ltd., Tokyo, 2015 (in Japanese).

2. Vestergaard P, Schwartz K, Pinholt EM, Rejnmark L, Mosekilde L. Gastric and esophagus events before and during treatment of osteoporosis. Calcif Tissue Int 86: 110-115, 2010.

3. Peter CP, Handt LK, Smith SM, et al. Esophageal irritation due to alendronate sodium tablets. Dig Dis Sci Sep 43: 1998-2002, 1998.

4. Peter CP, Kindt MV, Majka JA. Comparative study of potential for bisphosphonates to damage gastric mucosa of rats. Dig Dis Sci May 43: 1009-1015, 1998.

5. Groen PC, Lubbe DF, Hirsch LJ, et al. Esophagitis associated with the use of alendronate. N Engl J Med 335: 1016-1021, 1996.

6. Kusano M, Shimoyama Y, Sugimoto S, et al. Development and evaluation of FSSG: frequency scale for the symptoms of GERD. J Gastroenterol 39: 888-891, 2004.

7. Armstrong D, Benett JR, Blum AL, et al. The endoscopic assessment of esophagitis. A progress report on observer agreement. Gastroenterology 111: 85-92, 1996.

8. Kimura K, Takemoto T. Endoscopic recognition of the atrophic border and its significance in chronic gastritis. Endoscopy 3: 8797, 1969.

9. Makuuchi H. Clinical study of esophageal hiatal hernia - diagnostic criteria and degree classification of hiatal hernia-. Jpn J Gastroenterol 79: 1557-1567, 1982 (in Japanese, Abstract in English).

10. Sakita T, Oguro Y, Miwa T. Gastric lesions - gastric ulcers (including scars). In: Practical Training in Gastroenterological Endoscopy. Chugaiigaku Co., Ltd., Tokyo, 1981: 376-396 (in Japanese).

11. Takeshita E, Sakata Y, Hara M, et al. Higher frequency of reflux symptoms and acid-related dyspepsia in women than men regardless of endoscopic esophagitis: analysis of 3505 Japanese subjects undergoing medical health checkups. Digestion 93: 266-271, 2016.

12. Yamamichi N, Mochizuki S, Asada-Hirayama I, et al. Lifestyle factors affecting gastroesophageal reflux disease symptoms: a cross-sectional study of healthy 19864 adults using FSSG scores. BMC Medicine 10: 45, 2012.

13. Matsuki N, Fujita T, Watanabe N, et al. Lifestyle factors associated with gastroesophageal reflux disease in Japanese population. J Gastroenterol 48: 340-349, 2013.

14. Okamoto K, Iwakiri R, Mori M, et al. Clinical symptoms in endoscopic reflux esophagitis: evaluation in 8301 adult subjects. Dig
Dis Sci 48: 2237-2241, 2003.

15. Kishimoto H, Fukunaga M, Kushida K, et al. Efficacy and tolerability of once-weekly administration of $17.5 \mathrm{mg}$ risedronate in Japanese patients with involutional osteoporosis: a comparison with $2.5 \mathrm{mg}$ once-daily dosage regimen. J Bone Miner Metab 24: 405-413, 2006.

16. Hagino $\mathrm{H}$, Kishimoto $\mathrm{H}$, Ohishi $\mathrm{H}$, et al. Efficacy, tolerability and safety of once-monthly administration of $75 \mathrm{mg}$ risedronate in Japanese patients with involutional osteoporosis: a comparison with a $2.5 \mathrm{mg}$ once-daily dosage regimen. Bone 59: 44-52, 2014.

17. Miki T, Masaki H. Bisphosphonates and gastrointestinal adverse effects. Clin Calcium 19: 91-97, 2009 (in Japanese, Abstract in English).

18. Amagase K, Inaba A, Senta T. Gastric ulcerogenic and healing impairment effects of risedronate, a nitrogen-containing bisphosphonate in rats. Comparison with alendronate and minodronate. $\mathrm{J}$ Physiol Pharmacol 62: 609-618, 2011.

19. Lanza F, Schwartz H, Sahba B, et al. An endoscopic comparison of the effect of alendronate and risedronate on upper gastrointestinal mucosae. Am J Gastroenterol 95: 3112-3117, 2000.

20. Kim SH, Jeong JB, Kim JW, et al. Clinical and endoscopic characteristics of drug-induced esophagitis. World J Gastroenterol 20: 10994-10999, 2014.

21. Peng YL, Hu HY, Luo JC, et al. Alendronate, a bisphosphonate, increased upper and lower gastrointestinal bleeding: risk factor analysis from a nationwide population-based study. Osteoporosis 25: 1617-1623, 2014.

22. Kanatsu K, Aihara E, Okayama M, et al. Mucosal irritative and healing impairment action of risedronate in rat stomachs: comparison with alendronate. J Gastroenterol Hepatol 19: 512-520, 2004.

23. Blank MA, Gibson GW, Myers WR, et al. Gastric damage in the rat with nitrogen-containing bisphosphonates depends on PH. Aliment Pharmacol Ther 14: 1215-1223, 2000.

The Internal Medicine is an Open Access journal distributed under the Creative Commons Attribution-NonCommercial-NoDerivatives 4.0 International License. To view the details of this license, please visit (https://creativecommons.org/licenses/ by-nc-nd/4.0/).

(C) 2019 The Japanese Society of Internal Medicine Intern Med 58: 1049-1056, 2019 